\title{
SAÚDE MENTAL FEMININA E CICLO REPRODUTIVO: UMA REVISÃO DE LITERATURA
}

FEMALE MENTAL HEALTH AND REPRODUCTIVE CYCLE: A LITERATURE REVIEW

\author{
SALUD MENTAL FEMENINA Y CICLO REPRODUCTIVO: \\ UNA REVISIÓN DE LA LITERATURA
}

ANA CAROLINA
CERQUEIRA MEDRADO

MÔNICA LIMA ${ }^{1}$

${ }^{1}$ Universidade Federal da Bahia, Salvador/BA, Brasil
RESUMO: Neste artigo objetivamos refletir criticamente sobre a produção na literatura a respeito do adoecimento mental de mulheres em relação ao ciclo reprodutivo feminino, com destaque para marcadores sociais como raça e classe social. Trata-se de um recorte da revisão de literaura de uma tese que selecionou 80 artigos, correspondente à categoria "ciclo reprodutivo", totalizando 22 artigos. Na introdução fazemos uma retomada histórica da associação do ciclo reprodutivo à saúde mental feminina, advinda de uma leitura essencialista da mulher e da medicalização de seu corpo. Como referencial teórico, adotamos a Psicologia Social Construcionista e o Feminismo Interseccional. A pesquisa foi realizada na Biblioteca Virtual em Saúde e caracteriza-se como revisão de literatura integrativa. Na seleção consideramos artigos em inglês, português e espanhol no período entre 2014 a 2018. Os textos foram divididos em subtemas relacionados ao objetivo do artigo, considerando o transtorno mental e algum período do ciclo reprodutivo feminino. Os achados da revisão enfocam os determinantes sociais envolvidos no adoecimento feminino, como pobreza, sobrecarga de trabalho e violência. Concluímos que as informações do estudo permitem desessencializar 0 adoecimento mental feminino, indicando que as atribuições sociais às mulheres têm sido fonte de adoecimento para elas.

Palavras-chave: mulher; saúde mental; Psicologia Social Construcionista; Feminismo Interseccional.

ABSTRACT: This article aims to reflect critically on the production in the literature regarding the mental illness of women in relation to the female reproductive cycle, with emphasis on social markers such as race and social class. It is an excerpt from the literature review of a thesis that selected 80 articles, corresponding to the category "reproductive cycle", totaling 22 articles. In the introduction, we make a historical return to the association of the reproductive cycle with female mental health, arising from an essentialist reading of women and the medicalization of their bodies. As a theoretical framework, we adopted Social Constructionist Psychology and Intersectional Feminism. The research was carried out at the Virtual Health Library and is characterized as an integrative literature review. In the selection, we considered articles in English, Portuguese and Spanish from 2014 to 2018. The texts were divided into sub-themes related to the objective of the article, considering the mental disorder and some period of the female reproductive cycle. The review's findings focus on the social determinants involved in female illness, such as poverty, work overload and violence. We conclude that the study information allows to de-essentialize female mental illness, indicating that the social attributions to women have been a source of illness for them.

Keywords: woman; mental health; medicalization; Social Constructionist Psychology; Intersectional Feminism.

RESUMEN: Este artículo tiene como objetivo reflexionar críticamente sobre la producción en la literatura sobre la enfermedad mental de las mujeres en relación con el ciclo reproductivo femenino, con énfasis en los marcadores sociales como la raza y la clase social. Es un extracto de la revisión de la literatura de una tesis que seleccionó 80 artículos, correspondiente a la categoría "ciclo reproductivo", totalizando 22 artículos. En la introducción, hacemos una reanudación histórica de la asociación del ciclo reproductivo con la salud mental femenina, que surge de una lectura esencialista de las mujeres y la medicalización de sus cuerpos. Como marco teórico, adoptamos la psicología social construccionista y el feminismo interseccional. La investigación se llevó a cabo en la Biblioteca Virtual en Salud y se caracteriza por ser una revisión bibliográfica integradora. En la selección consideramos artículos en inglés, portugués y español en el período comprendido entre 2014 y 2018. Los textos se dividieron en subtemas relacionados con el objetivo del artículo, considerando el trastorno mental y algún período del ciclo reproductivo femenino. Los resultados de la revisión se centran en los determinantes sociales involucrados en la enfermedad femenina, como la pobreza, la sobrecarga de trabajo y la violencia. Llegamos a la conclusión de que la información del estudio permite desesencializar la enfermedad mental femenina, lo que indica que las atribuciones sociales a las mujeres han sido una fuente de enfermedad para ellas.

Palabras clave: mujer; salud mental; medicalización; Psicología Social Construccionista; Feminismo Interseccional.
Recebido em 28/03/2020 Aprovado em 03/07/2020 
A articulação entre ciclo reprodutivo e saúde mental feminina remonta aos primórdios da medicina e da psiquiatria, momento no qual a doença mental feminina era localizada dentro do corpo da mulher e, mais especificamente, em seus órgãos reprodutores/sexuais. Assim, era comum intervenções como extirpações do clitóris, cirurgias no útero etc. para um suposto restabelecimento da saúde. Irregularidades menstruais, desejo sexual "excessivo" e o descumprimento de seu "destino natural" como mãe podiam ser vistos tanto como causa quanto como sintoma de doença mental, haja vista que a doença mental era encarada como um desvio moral. Destarte, medicalizava-se o corpo feminino, fazendo dele campo de experimentações de uma medicina misógina aliada ao discurso religioso (Del Priore, 2008; Engel, 2008). Dessa maneira, a medicalização do corpo feminino se inicia, justamente, pela via do que hoje compreendemos como saúde reprodutiva/sexual.

Partimos da noção de que "[...] medicalizar significa o processo de transformar aspectos da vida cotidiana em objetos da medicina de forma a assegurar conformidade às normas sociais" (Miles, 1991 citado por Vieira, 1999, p. 68). Por exemplo, o parto, antes visto como atividade feminina, assumido pelas parteiras, cada vez mais será circunscrito no saber médico. Antes sob a alçada do saber popular, o parto passa a ser progressivamente dominado por especialistas masculinos com a exigência de licença para exercer a atividade e com a perseguição às parteiras (vistas como bruxas pela Inquisição Católica, sobretudo no período entre 1563 e 1727). Tal processo foi dando outras feições ao parto: o saber popular era agora preterido e a instrumentalização do parto se expande. Ademais, um fenômeno outrora natural era agora "tratável" pela medicina (Spink, 2003; Vieira, 1999).

Entretanto, é válido ressaltar que o acesso ao médico para realização do parto não se deu de maneira uniforme. A princípio os médicos assumiam, principalmente, os partos mais complicados e suas intervenções eram rejeitadas, pois apresentavam alta taxa de mortalidade. Mesmo quando a influência médica passou a ser mais corriqueira, o acesso a um parto hospitalar - com uso de fórceps, anestesia etc. - era restrito às mulheres ricas e do espaço urbano, visto como sinal de ostentação. Dessa maneira, é possível delinear duas histórias do parto, atravessadas pelas questões de classe social (Spink, 2003; Vieira, 1999). Como ratifica Mary Jane Spink (2003), referente à saúde reprodutiva, as mulheres pobres eram mais utilizadas como sujeitos de pesquisa do que beneficiárias da prática médica. No tocante a isso, destacamos os testes de James Marion Sims, "pai da ginecologia moderna", que fazia cirurgias em mulheres negras escravizadas, muitas vezes sem o uso de anestesia, de acordo com Juliana Lima (2016).

Nessa mesma direção, sob domínio do saber masculino atrelado a valores morais, o corpo feminino era visto, de acordo com Mary Del Priore (2008), como desviante, "como um palco nebuloso e obscuro no qual Deus e o Diabo se digladiavam" (p. 78). Sendo o corpo masculino a norma, o corpo feminino era encarado com suspeição. O sangue menstrual, por sua vez, possuía poderes mágicos, podendo estragar o vinho, a colheita e o leite. A mulher saudável era aquela que procriava e, dessa maneira, conseguia escapar dos males provenientes do útero (Del Priore, 2008). Assim, a medicalização do corpo feminino ocorreu em sintonia com uma visão essencialista da mulher, com a ideia de "natureza feminina". O "ser mulher" era tido como determinado pela biologia, pela capacidade feminina de parir, amamentar, menstruar; o que se reflete no "instinto maternal" e nas atividades de cuidado assumidas pelas mulheres, vistos como naturais, conforme Elisabeth Vieira (1999).

A medicalização do corpo feminino e a medicalização da loucura são temas que se interpenetram. Segundo Magali Engel (2008), as práticas psiquiátricas no Brasil do século XIX, por exemplo, tinham compromisso com a ordem social, tendo como 
uma das principais intervenções as condutas individuais, o comportamento no trabalho, a segurança e os comportamentos sexuais, sendo as mulheres os alvos basais: uma sexualidade considerada anormal, ou seja, aquela excessiva ou deficitária nas relações conjugais, poderia ser sintoma de histeria. Outros "desvios" tais como falta de afeição ao marido e ao filho e desinteresse pelas atividades domésticas também poderiam ser sintomas de doença mental. Isso porque a mulher era relacionada à natureza, ao passo que o homem era identificado com a cultura de maneira tal, que as doenças mentais nas mulheres eram aquelas relativas à sua "natureza", sobretudo à sua sexualidade; enquanto aos homens eram atribuídos sintomas ligados à cultura, como o desinteresse pelo trabalho, por exemplo (Engel, 2008).

Entretanto, a relação entre ciclo reprodutivo e transtorno mental não é algo que ficou no passado. Atualmente, ao consultarmos a quinta edição do Manual Diagnóstico e Estatístico (DSM-V, 2014), encontramos entre os diagnósticos o Transtorno Disfórico Pré-menstrual, classificado entre os transtornos depressivos, que se caracteriza por: "[...] expressão de labilidade do humor, irritabilidade, disforia e sintomas de ansiedade que ocorrem repetidamente durante a fase pré-menstrual do ciclo e remitem por volta do início da menstruação ou logo depois" (DSM-V, 2014, p. 172).

Neste artigo temos como objetivo refletir criticamente sobre a produção na literatura a respeito do adoecimento mental de mulheres em relação ao ciclo reprodutivo feminino, com destaque para marcadores sociais como raça e classe social. Para tanto, utilizamos alguns aportes da Psicologia Social Construcionista (PSC), articulada ao Feminismo Intersecccional.

O construcionismo tem suas raízes em movimentos (no campo das ciências humanas e sociais) que refutam a neutralidade da produção científica e a assunção da verdade. Uma das críticas à ciência hegemônica refere-se, justamente, ao seu caráter machista e racista, encoberto sob um véu de suposta imparcialidade (Gergen, 2011). A PSC rejeita o essencialismo e a ênfase no indivíduo, advogando pela compreensão de construções sociais, histórica e culturalmente situadas, tanto no que se refere à identidade, quanto à produção de conhecimento. Enfatizando a linguagem, encarada como uma prática social, a PSC rejeita a ideia de um eu ensimesmado, de uma estrutura interna que contém o eu. Assim, Kenneth Gergen (2011) afirma que o eu, para a PSC, é um eu discursivo, o que é válido também para a identidade de gênero.

Segundo Conceição Nogueira (2001), a PSC alinha-se ao feminismo de terceira onda, que nega as essencializações e objetiva analisar outras opressões que interagem com o gênero, tais como as de classe social, raça, orientação sexual e religião, em uma proposta interseccional. Nogueira (2017) assevera que a teoria interseccional permite investigar as identidades de maneira complexa, multiplicativa, e também dinâmica, variando de acordo com as interações sociais.

\section{MÉTODO}

Este artigo é um recorte da revisão de literatura integrativa que compõe a tese intitulada "Cuidado e sofrimento mental feminino: sentidos construídos pelas profissionais de saúde", em andamento. Este tipo de revisão é composta das seguintes etapas: 1) identificação do tema e seleção da questão de pesquisa, que inclui uma análise da adequação de descritores e escolha de bases indexadoras; 2) estabelecimento de critérios de inclusão e exclusão; identificação dos estudos pré-selecionados e selecionados, realizada com a leitura do título, resumo e das 
palavras-chaves, se necessário incluindo a leitura do artigo na íntegra; 4) categorização dos estudos selecionados, que pode destacar o tamanho da amostra, quantidade de sujeitos, métodos de coleta e análise e teoria ou conceitos que embasam o estudos; 5) análise e interpretação dos resultados, quando se levantam as lacunas; 6) apresentação da revisão ou síntese do conhecimento, como definem Louise Botelho, Cristiano Cunha e Marcelo Macedo (2011).

$\mathrm{Na}$ revisão original que compõe a referida tese tivemos como questão: quais modelos explicativos estão construídos em torno do adoecimento mental das mulheres na produção científica disponível na Biblioteca Virtual em Saúde (BVS)? A BVS é uma das bases de acesso livre mais completa no campo da saúde, o que justifica nossa escolha por ela. A coleta e dados foi realizada no dia 24 de agosto de 2018, fazendo uso dos descritores woman e mental health (Descritores em Ciências Sociais da Bibilioteca Virtual em Saúde), com o operador booleano and.

A procura resultou em um total de quatro mil seiscentos e setenta e três (4673) escritos, por isso foi necessário realizar uma série de refinamentos: definimos o período de cinco anos de publicação dos textos (2014-2018); restringimos a língua ao português, inglês e espanhol; excluímos aqueles que não tiveram revisão cega por pares e aqueles que não tinham acesso aberto. Essa etapa resultou em oitocentos e cinquenta e dois textos (852). A etapa seguinte foi selecioná-los a partir do título. Apesar do uso dos descritores e do operador booleano ainda foram capturadas produções que não abrangiam a nossa temática. Dessa forma, consideramos como fugindo do tema um total de quinhentos e cinquenta e três (553) escritos (respeitando também os critérios de exclusão adotados, como ser artigo de revisão, por exemplo). Nessa segunda fase de refinamento também foram excluídos: os artigos que não são de acesso aberto (162), os trabalhos repetidos (10), um (01) artigo retratado (artigo que apresenta má conduta científica como plágio, fabricação de informações etc.) e um (01) artigo não encontrado na íntegra. Assim, após leitura do título, restaram cento e vinte e cinco (125) produções. O próximo passo foi a leitura dos resumos. Considerando o grande quantitativo de títulos lidos, nessa etapa ainda foi possível encontrar textos que fugiam ao tema e que não abordavam especificamente modelos explicativos ou agravantes de transtorno mental nas mulheres, o que culminou na exclusão de quarenta e cinco (45) deles. Por fim, restaram como estudos de interesse 80 produções.

Os 80 artigos foram organizados em tabelas, destacando em colunas autoria/ ano, país de origem do estudo, objetivo do artigo, metodologia adotada, resultados e categoria temática. As categorias foram definidas por meio do uso do critério de analogia considerando o objetivo do artigo de referência. Este processo envolveu três etapas: 1. Pré-análise; 2. Codificação; e 3. Categorização (Vázquez Sixto, 1996). Contudo, entendemos que às vezes é possível inscrever um artigo em mais de uma categoria devido ao objetivo abordar mais de um tema. Em tais casos, foi levada em conta a temática que foi mais discutida no artigo.

Assim, os resultados gerais da revisão dos 80 itens foram organizados em 10 categorias temáticas, sendo uma delas denominada como "ciclo reprodutivo", na qual inserimos os estudos que relacionam o transtorno mental em mulheres a algum período do ciclo reprodutivo feminino, artigos que serão analisados nesta revisão. A referida categoria obteve 22 produções, representando $27,5 \%$ dos resultados da revisão de literatura completa, sendo a que condensou mais estudos. O fato de esta categoria específica entre outras dez condensar tantos estudos nos chamou atenção por reportar aos primórdios da psiquiatria, conforme discutido na introdução do texto. Remete-nos também a uma redução da mulher à sua capacidade sexual/reprodutiva. A categoria foi dividida nos subtemas 
seguintes: a) depressão/maternity blues e/ou ansiedade gestacional e/ou pós-parto; b) transtorno mental comum (TMC) em gestantes e/ou no pós-parto;c) má-formação congênita do bebê e efeitos sobre a saúde mental da mãe; d) perda gestacional e consequências na gestação subsequente; e) sintomas psiquiátricos não psicóticos em gestantes;f) depressão no pré-climatério; g) efeitos da pré-menstruação, gestação, pré-menopausa, da dispareunia e da dor pélvica na saúde mental; h) ideação/ comportamento suicida entre gestantes; i) queixas de saúde mental que levam as gestantes a procurar o serviço de saúde (Tabela 1). A classificação dos artigos nos referidos subtemas se deu considerando os objetivos de cada produção, seguindo o mesmo critério da revisão mais ampla que compõe a tese. Acreditamos ser válido recorrer a tal divisão pois é possível explicitar melhor os diferentes transtornos mentais e períodos do ciclo reprodutivo presentes na referida categoria.

Tabela 1: Subtemas, quantidade e referências bibliográficas da categoria "ciclo reprodutivo" em artigos da BVS, entre 2014 e 2018

\begin{tabular}{|c|c|c|}
\hline Subtemas & $\begin{array}{l}\text { Quantidade } \\
\text { de textos/ } \\
\text { percentual }\end{array}$ & Referências bibliográficas \\
\hline $\begin{array}{c}\text { Depressão/maternity blues e/ou } \\
\text { ansiedade gestacional e/ou pós-parto }\end{array}$ & $\begin{array}{c}12 \\
54,6 \%\end{array}$ & $\begin{array}{l}\text { Chojenta, Lucke, Forder e Loxton, 2016; } \\
\text { Ghaffar et al., 2017; Kang et al., 2016; Kathree, } \\
\text { Selohilwe, Bhana e Petersen, 2014; Moraes } \\
\text { Costa, Silva, Silva e Melo, 2017; Moura, Pedrão, } \\
\text { Souza e Boaventura, 2015; Ogbo et al., 2018; } \\
\text { Takahashi e Tamakoshi, 2014; Van Vo, Hoa e } \\
\text { Hoang, 2017; Verbeek, Arjadi, Vendrik, Burger } \\
\text { e Berger, 2015; Wang et al., 2016; Woolhouse, } \\
\text { Gartland, Mensah e Brown, } 2014 .\end{array}$ \\
\hline $\begin{array}{l}\text { Transtorno mental comum (TMC) } \\
\text { em gestantes e/ou no pós-parto }\end{array}$ & $\begin{array}{c}03 \\
13,7 \%\end{array}$ & $\begin{array}{l}\text { Abrams et al., 2016; Lucchese et al., 2017; } \\
\text { Nguyen et al., } 2015 .\end{array}$ \\
\hline $\begin{array}{l}\text { Má-formação congênita do bebê } \\
\text { e efeitos sobre a saúde mental da mãe }\end{array}$ & $\begin{array}{c}01 \\
4,52 \%\end{array}$ & $\begin{array}{c}\text { Cunha, Pereira Junior, } \\
\text { Caldeira e Carneiro, } 2016 .\end{array}$ \\
\hline $\begin{array}{l}\text { Perda gestacional e consequências } \\
\text { na gestação subsequente }\end{array}$ & $\begin{array}{c}01 \\
4,52 \%\end{array}$ & Chojenta et al., 2014. \\
\hline $\begin{array}{l}\text { Sintomas psiquiátricos } \\
\text { não psicóticos em gestantes }\end{array}$ & $\begin{array}{c}01 \\
4,52 \%\end{array}$ & $\begin{array}{l}\text { Reis, Bernardes, Barboza } \\
\quad \text { e Gonçalves, } 2015 .\end{array}$ \\
\hline Depressão no pré-climatério & $\begin{array}{c}01 \\
4,52 \%\end{array}$ & $\begin{array}{l}\text { Guadalupe, López Jiménez } \\
\text { e García González, } 2017 .\end{array}$ \\
\hline $\begin{array}{l}\text { Efeitos da pré-menstruação, gestação, } \\
\text { pré-menopausa, da dispareunia e da } \\
\text { dor pélvica na saúde mental }\end{array}$ & $\begin{array}{c}01 \\
4,52 \%\end{array}$ & Miller e Ghadiali, 2015. \\
\hline $\begin{array}{l}\text { Ideação/comportamento } \\
\text { suicida entre gestantes }\end{array}$ & $\begin{array}{c}01 \\
4,52 \%\end{array}$ & $\begin{array}{l}\text { Onah, Field, Bantjes } \\
\text { e Honikman, } 2017 .\end{array}$ \\
\hline $\begin{array}{l}\text { Queixas de saúde mental que levam as } \\
\text { gestantes a procurar o serviço de saúde }\end{array}$ & $\begin{array}{c}01 \\
4,52 \%\end{array}$ & Tol et al., 2018. \\
\hline
\end{tabular}

Nova Perspectiva Sistêmica, v. 29, n. 67, p. 70-84, agosto 2020. 


\section{RESULTADOS}

Os artigos capturados em nossa revisão apresentaram uma diversidade de países de realização: Brasil (05), Austrália (04), Vietnã (03), África do Sul (02), China (02), Japão (01), Uganda (01), Paquistão (01), Nicarágua (01), México (01), Estados Unidos (01). Contudo, o desenho de estudo adotado nas pesquisas foi bem mais homogêneo: a maioria foi desenvolvida no campo epidemiológico, sendo 13 estudos de corte transversal e 06 de coorte.

A despeito da diversidade de países que compõem os estudos da presente revisão, não foi possível verificar muitas discussões sobre especificidades culturais nos referidos textos, ficando a marca característica da generalização dos estudos quantitativos, o que reduz a diversidade cultural e torna muitos dos estudos apenas uma replicação de outrem em país diferente. Isso fica mais premente quando atentamos aos instrumentos (escalas, inventários etc.). Quando enfocamos os estudos sobre depressão gestacional e depressão pós-parto, por exemplo, foi corrente o uso da Edinburgh Postnatal Depression Scale (EPDS) em contextos díspares como Japão e África do Sul. O uso dos mesmos instrumentos validados para diferentes contextos culturais é um valor dentro dos estudos quantitativos, mas, por outro lado, quando homogeneizamos as idiossincrasias culturais, tanto faz dizer que a pesquisa se passou no Paquistão ou no Brasil.

Por conta disso, e entendendo que uma revisão pode apresentar seus resultados de inúmeras maneiras, o referencial teórico adotado ajudou na seleção de quais artigos seriam discutidos e na ênfase que seria dada a determinadas informações como diferenças culturais e raciais, por exemplo. Assim, procuramos dar destaque a textos em que pudéssemos fugir a uma leitura individualizante, essencialista e internalista (muitas vezes indo além do que é apresentado apenas como "dado" na seção de resultados dos artigos), e que trouxessem outras informações que cremos mais relevantes do que meras estatísticas relativas ao adoecimento feminino. Nessa tônica, em uma articulação da Psicologia Social Construcionista e do Feminismo Interseccional, a nossa seleção dos textos que serão discutidos mais profundamente aqui, visto não ser possível discorrer sobre a totalidade dos resultados (22 artigos), visa marcar que quando nos referirmos à mulher e ao feminino, não buscamos universalizar ou cristalizar tais termos. Nesse sentido, destacamos os resultados dos artigos mais emblemáticos para cada subtema discutido.

O subtema "Depressão/maternity blues e/ou ansiedade gestacional e/ou pós-parto" concentrou a maior parte das produções da revisão, totalizando 12 artigos, o que corresponde a aproximadamente $54,5 \%$ do total. Nesse subtema, alguns estudos nos indicam que as diferenças socioeconômicas e culturais podem estar envolvidas na experiência depressiva e/ou ansiosa. É o caso do estudo de Verbeek et al. (2015), que investigaram a prevalência e severidade de ansiedade e depressão durante a gestação e a acessibilidade aos serviços de saúde mental na Nicarágua, em comparação com a Holanda. O estudo concluiu que $41 \%$ das mulheres nicaraguenses apresentaram ao menos sintomas moderados de ansiedade e $57 \%$ ao menos sintomas moderados de depressão, enquanto entre as holandesas as taxas foram bem menores, com 15\% e $6 \%$, respectivamente. Entretanto, apenas $9,6 \%$ das nicaraguenses recebiam ajuda psicológica. Os dados indicam que a prevalência de ansiedade e depressão em gestantes nicaraguenses é alta em comparação com países desenvolvidos. É válido ressaltar que apenas $1 \%$ da verba de saúde na Nicarágua é investida na saúde mental e, de tal verba, $91 \%$ é investida em hospitais psiquiátricos.

Outra pesquisa relevante quanto à diversidade cultural foi a realizada por Yuki Takahashi e Koji Tamakoshi (2014), no Japão, que descobriram que a prática cultural 
satogaeri buben (tradição japonesa de suporte à mãe nos primeiros dias após o parto em que ela retorna à casa dos pais) foi fator protetivo da saúde mental da mulher no pós-parto em relação ao maternity blues.

Ainda em relação à cultura, na China e no Vietnã, por exemplo, os bebês do sexo masculino são mais desejados, o que pode fazer com que as mães fiquem mais ansiosas durante a gestação, de acordo com Yu-tin Kang et al. (2016) e Thang Van Vo et al. (2017). Nessa direção, estudo realizado no Vietnã verificou que as mulheres que geraram bebês com o sexo diferente do desejado tiveram maior prevalência de sintomas depressivos quando comparadas com aquelas com o sexo conforme esperado, embora o resultado não tenha se mantido após regressão logística multivariada (Van Vo et al., 2017).

Felix Ogbo et al. (2018) também encontraram influência da diversidade cultural, bem como socioeconômica, relacionada à depressão. Em estudo realizado na Austrália que incluía mulheres com diversidades culturais e linguísticas, tais mulheres apresentaram maior prevalência de sintomas depressivos que o grupo mais homogêneo quanto a língua e cultura. Ademais, as mães de baixa renda tiveram mais chances de apresentar depressão pós-parto que as mães de alta renda. Tasneem Kathree et al. (2014) e Valéria Moura et al. (2015) também mencionam a pobreza como relacionada ao adoecimento. Moura et al. (2015) ainda relataram ser negra como fator de risco para a depressão, sendo que $40 \%$ das negras apresentaram depressão e nenhuma gestante branca apresentou sintomatologia depressiva nesse estudo local.

Outro subtema de nossa revisão é "Transtorno mental comum (TMC) em gestantes e/ou no pós-parto", que apresentou três achados. Referente ao subtema, Trang Nguyen et al. (2015) conduziram pesquisa para investigar fatores de risco e proteção ao TMC no pós-parto entre mulheres da zona rural do Vietnã. Os resultados apontaram que a incidência de TMC nas mulheres no primeiro ano pós-parto foi de $13 \%$ e foi associada com pouca ajuda e pouca atenção do marido, maus-tratos na infância e pobreza. Quanto à recuperação do TMC, as mulheres que tiveram ao menos um mês de cuidados de outrem após o parto, antes de retomar à rotina, tiveram maior probabilidade de recuperação. Aquelas que não experienciaram violência doméstica e que tinham ajuda nas tarefas da casa e cuidados com o/a bebê também tiveram maior propensão para recuperação.

Ainda no Vietnã, Daniel Abrams et al. (2016) realizaram pesquisa qualitativa em quatro comunidades rurais com minorias étnicas. As entrevistas foram conduzidas tendo dois casos como mote (um caso de depressão pós-parto e outro de ansiedade generalizada). Foram entrevistadas mulheres grávidas ou com um ano de pós-parto e profissionais de saúde. As/os participantes deveriam opinar sobre os casos em tela. Entre os aspectos que emergiram na entrevista, destacamos a menção de fitoterápicos vistos como importantes para alimentação da gestante na manutenção de sua saúde, bem como medicamentos fitoterápicos foram considerados mais adequados para os casos de mulheres gestantes ou lactantes com algum tipo de transtorno mental, por apresentarem menos efeitos colaterais. Entre as/os profissionais (total de 12), apenas um/a recebeu algum tipo de formação em saúde mental. Muitas/ os profissionais afirmaram que tais casos eram encaminhados para hospitais por serem mais especializados.

A terceira investigação do subtema foi conduzida no Brasil. Roselma Lucchese et al. (2017) encontraram alta prevalência de TMC entre as gestantes brasileiras em comparação com a população geral de 63 países, com taxa de 57,1\%. As/os pesquisadoras/es encontraram maiores chances de apresentar TMC entre as mulheres de renda familiar mais alta (maior que $\mathrm{R} \$ 1.581,09$ ). 
Outro subtema investigado foi "Ideação/comportamento suicida entre gestantes", com trabalho desenvolvido por Michael Onah et al. (2017), que realizaram a investigação na área mais violenta da Cidade do Cabo, África do Sul. Os resultados foram: a prevalência de SIB (suicidal ideation and behaviours) foi de 18\%; nove mulheres tinham tentado suicídio no último mês, dentre elas, cinco com a intenção de morrer; dentre as mulheres que apresentaram SIB, 33\% tinham depressão maior, $35 \%$ algum tipo de transtorno ansioso e $54 \%$ nenhum dos dois transtornos. Tanto a depressão maior como o transtorno ansioso foram associados com a SIB. Aquelas que tinham suporte social tinham menos chances de SIB. As mulheres solteiras tiveram mais chances de ter SIB e as que sofreram violência do parceiro tinham o dobro de chances daquelas que não sofreram. As mulheres com insegurança alimentar (déficit na quantidade e qualidade do alimento) tinham quatro vezes mais chances de comportamento suicida que aquelas com segurança alimentar. A pobreza também esteve associada ao comportamento suicida.

Quanto ao subtema "Queixas de saúde mental que levam as gestantes a procurar o serviço de saúde", percebemos que as diferenças culturais podem também influenciar a maneira como o transtorno mental é percebido e classificado. Sobre isso, Wietse Tol et al. (2018), em estudo qualitativo, descobriram que, em Uganda, um dos motivos relativos à saúde mental que faz com que as mulheres grávidas procurem o serviço de saúde foi o que é conhecido como adeka na aomisio (doença dos pensamentos) e foi considerado um entre os três maiores problemas de saúde mental. Outros dois problemas mencionados foram malária e epilepsia. As/os especialistas em saúde mental que participaram da pesquisa se referiram ao adeka na aomisio como maternity blues. As mulheres gestantes afirmaram que os pensamentos tinham vida própria, sendo que elas não tinham controle sobre eles. Os sintomas atribuídos ao adeka na aomisio e maternity blues foram: tristeza e choro excessivos, mau humor, distúrbios do sono, anedonia, pensamentos suicidas, falta de apetite, irritabilidade, uso de substâncias, fobias etc. Participantes ainda citaram febre, alucinações e fala descoordenada. A "doença dos pensamentos" foi atribuída à ausência de companheiro ou de suporte por parte desse, bem como gravidez não desejada, pobreza e violência doméstica.

Entre os estudos que se afastam das discussões que enfatizam os fatores psicossociais quanto ao adoecimento, se aproximando de uma leitura mais biológica quanto ao adoecimento mental, destacamos o de Laura Miller e Nafisa Ghadiali (2015) no subtema "Efeitos da pré-menstruação, gestação, pré-menopausa, da dispareunia e da dor pélvica na saúde mental”, que avaliaram tanto a relação entre o ciclo reprodutivo como condições ginecológicas que causam dor (dispareunia e dor pélvica) e a saúde mental em veteranas. Foram estudadas 68 mulheres com média de 41 anos, a maioria delas ainda menstruava. Todas as mulheres apresentaram algum transtorno mental (o campo de estudo foi um serviço psiquiátrico): 30,9\% apresentaram transtorno do estresse pós-traumático; 29,4\% depressão; e 14,7\% transtornos de ansiedade. A taxa de hospitalização psiquiátrica foi de $27,9 \%$ e 20,6\% tentaram suicídio. Os resultados ainda demonstram que: $22,1 \%$ tinham dispareunia; $17,6 \%$ dor pélvica; $42,6 \%$ das mulheres acreditavam que a prémenstruação influenciava em seus problemas emocionais e psiquiátricos, a gravidez tinha influência para $33,3 \%$, bem como o pós-parto, e 18,2\% atribuíram influência negativa do período pré-menopausa na saúde mental.

A extensão de resultados agregados na nossa revisão não permitiu que todos os estudos fossem apresentados com minúcia. Mas, em síntese, os achados indicam algum tipo de associação entre transtorno mental (transtorno mental comum, depressão, transtorno ansioso, ideação e/ou comportamento suicida) na gestação 
ou pós-parto e os seguintes fatores mais recorrentes (ao menos em três estudos): gravidez não planejada (Kathree et al., 2014; Lucchese et al., 2017; Tol et al., 2018; Van Vo et al. 2017); não ter ajuda do companheiro (Nguyen et al., 2015; Tol et al., 2018; Van Vo et al., 2017); pobreza (Kathree et al., 2014; Moura et al., 2015; Nguyen et al., 2015; Ogbo et al., 2018; Onah et al., 2017; Tol et al., 2018); violência por parte do parceiro (Kathree et al., 2014; Ogbo et al., 2018; Onah et al., 2017; Tol et al., 2018).

\section{DISCUSSÃO}

Discutindo os resultados referentes à raça/cor, no estudo de Moura et al. (2015), realizado no Brasil, podemos encontrar relação com o efeito do racismo na saúde mental. Segundo Rita Barata (2009), dentre os problemas de saúde associados ao racismo e à discriminação estão os transtornos mentais. As vítimas de discriminação (sendo o racismo uma das mais relevantes) têm maior prevalência de distúrbios do sono, depressão, ansiedade, ideação e tentativa de suicídio. Sofrer discriminação também implica menor probabilidade de procurar os serviços de saúde (Barata, 2009). Se promovermos a intersecção de outras discriminações, podemos pressupor vulnerabilidades em saúde ainda mais salientes. Nesse sentido, concordamos com Emanuelle Goes e Enilda Nascimento (2013) quando afirmam que:

A desigualdade não decorre da diferença individual, mas do modo como as pessoas estão organizadas socialmente, em uma estrutura hierarquizada por valores simbólicos e materiais produzida pelas variadas relações sociais e que tem como consequência a repartição não uniforme de todos os tipos de vantagens e desvantagens. As desigualdades sociais são, portanto, resultado de processos sociais, demográficos, econômicos, culturais e políticos desenvolvidos em contextos determinados social e historicamente. (p. 572)

As desigualdades sociais também se expressam em relação ao TMC. Concernente a isso, o resultado de Lucchese et al. (2017), quanto à renda familiar maior associada ao TMC, contradiz as evidências sobre o transtorno: a prevalência de TMC tem sido associada aos fatores socioeconômicos como desigualdades de gênero, baixa renda, baixa escolaridade, desemprego/informalidade (Ludermir, 2008; Zanello, 2014). Sobre isso, Ana Ludermir (2008) afirma: "O núcleo essencial da concepção de causalidade da doença mental não se encontra numa determinada classe ou gênero, mas sim nas relações estabelecidas entre classes e gêneros, caracterizadas pela opressão e subordinação de um grupo por outro" (p. 461).

Enquanto Ludermir (2008) alerta para a dinamicidade das relações sociais participando das "causas" do transtorno mental, Valeska Zanello (2014) chama a atenção para uma possível patologização das mazelas sociais que pode estar envolvida no diagnóstico de TMC. Assim, percebemos que as mulheres em condições de pobreza estão mais vulneráveis ao TMC, mesmo que seja por conta de um processo de medicalização da vida, que provavelmente tem escamoteado os problemas sociais por meio de intervenções e diagnósticos médicos.

Referente ao subtema "Ideação/comportamento suicida entre gestantes", José Bertolote (2012) assevera que as diferenças de gênero também se expressam nas taxas de suicídio: o suicídio tem sido mais elevado entre os homens, enquanto a tentativa de suicídio é maior entre as mulheres. O autor credita tal diferença ao método empregado para cometer o ato: os homens tendem a fazer uso de recursos mais violentos (armas de fogo, por exemplo), que tem maior letalidade.

Nova Perspectiva Sistêmica, v. 29, n. 67, p. 70-84, agosto 2020. 
Essas informações são corroboradas na pesquisa de Felipe Baére e Valeska Zanello (2018) sobre suicídio e gênero no Distrito Federal. Os pesquisadores encontraram, a partir de dados epidemiológicos, que nos casos de óbito por suicídio por intoxicação as mulheres tiveram valores mais elevados frente os homens; já no caso do uso de arma de fogo, a situação se inverte, sendo a maioria realizada por homens. Ademais, os autores, fazendo uso de dados do boletim epidemiológico do Ministério da Saúde, ainda reafirmam a disparidade entre as tentativas de suicídio: 69\% entre as mulheres e $31 \%$ entre os homens. Entretanto, Bertolote (2012) identifica uma aproximação entre o índice de suicídio dos homens e das mulheres a partir de 19801990, o que ele suspeita que esteja relacionado a uma diminuição da desigualdade de gênero ou ao acréscimo dos dados de países africanos, sul-americanos e asiáticos, incluídos desde então.

Para além das questões de gênero, no estudo de Onah et al. (2017) as/os autoras/ es encontraram relação do suicídio com a insegurança alimentar e a pobreza. Sobre tal temática, Flávia Alves, Daiane Machado e Maurício Barreto (2018) realizaram um estudo ecológico sobre o efeito da cobertura do Programa Bolsa Família nas taxas de suicídio em 5507 municípios brasileiros e descobriram que uma alta cobertura do Programa, bem como uma cobertura por três ou mais anos, tinha como efeito uma redução nas taxas de suicídio. Tais investigações trazem subsídios para desassociarmos o suicídio de uma visão individualista e/ou essencialista em relação à pobreza e nos voltarmos para os determinantes sociais envolvidos na autoquíria e no delineamento de políticas públicas para sua prevenção.

Sobre a nossa temática, é válido discutir as duas interpretações dadas para as diferenças entre as prevalências de transtorno mental entre homens e mulheres (mulheres sofrem mais de depressão e transtorno de ansiedade, ao passo que os homens têm maior prevalência na adicção às drogas): uma biológica, que atribui a depressão feminina à influência do estrógeno, por exemplo; e outra sócio-histórica, que enfoca as "causas" sociais, inclusive as desigualdades de gênero, às quais nos alinhamos em nossa revisão (Zanello, 2014).

O expressivo quantitativo de textos que compõe este artigo, frente ao total de produções de nossa revisão completa, nos remete à relação entre a psiquiatria e o ciclo reprodutivo feminino no século XIX, conforme discutimos brevemente em nossa introdução. Contudo, seguindo a divisão proposta por Zanello (2014), percebemos que a maioria dos textos se atém à discussão sócio-histórica (como grau de escolaridade da mulher, renda da família, apoio do companheiro) envolvida no adoecimento mental feminino.

Nesse sentido, o único artigo que destoa de tal interpretação, dando ênfase a uma interpretação biológica, é o de Miller e Ghadiali (2015). Apesar de, no referido estudo, ter se considerado o autorrelato das mulheres quanto à percepção da influência do ciclo reprodutivo na saúde mental, é relevante discutir como um aspecto que é inerente à condição de ser mulher cisgênero (ter ciclo reprodutivo) foi associado como "causa" de adoecimento mental e, mais ainda, durante todas as fases de tal ciclo. Assim, percebe-se o corpo masculino sendo tomado como padrão de normalidade, o que, novamente, remonta à misoginia da psiquiatria do século XIX, e se remete ainda ao Transtorno Disfórico Pré-menstrual definido pelo DSM-V (2014).

A proposta de patologizar o ciclo reprodutivo presente no texto de Miller e Ghadiali (2015) e no DSM-V (2014) aproxima-se daquilo que Gergen (2010) identifica como espiral mórbido: as pessoas, por conta do discurso psiquiátrico corrente, sentem-se impelidas a reconhecerem-se dentro de um determinado rótulo diagnóstico o que, ao mesmo tempo, incentiva as/os profissionais psi a criarem um novo vocabulário para enquadrar ainda mais pessoas. Relembremos que o discurso

Nova Perspectiva Sistêmica, v. 29, n. 67, p. 70-84, agosto 2020. 
científico reproduzido pelas/os psiquiatras e psicólogas/os foi interagindo com o restante da sociedade de forma a moldar e normatizar as condutas.

Podemos considerar que o espiral mórbido reverbera no uso indiscriminado de benzodiazepínicos, uso muito mais expressivo entre as mulheres, conforme síntese realizada por Melissa Pereira e Paulo Amarante (2017). Ademais, a associação entre menopausa/climatério a transtornos mentais traz uma nova roupagem para a parceria entre ginecologia e psiquiatria: intervenções cirúrgicas como retirada de ovários, extirpação de clitóris, entre outras, tão comuns no século XIX, atualmente são substituídas por reposição hormonal. Assim, referente ao artigo de Miller e Ghadiali (2015), percebemos que, apesar do método de intervenção ter mudado, a interpretação sobre o adoecimento mental da mulher ainda permanece arraigada a uma visão biomédica, recaindo sobre os hormônios femininos.

\section{CONSIDERAÇÕES FINAIS}

A revisão de literatura permitiu desessencializar o adoecimento mental feminino, indicando que as atribuições sociais às mulheres têm sido fonte de adoecimento para elas. Procuramos refletir em nosso texto sobre as discussões dos fatores socioeconômicos, culturais e raciais, aliadas a uma análise da psicologia social construcionista e feminista interseccional do adoecimento mental feminino. Partindo de tal análise, as afirmações essencialistas caem em ruína, pois, na medida em que nos atemos ao desenvolvimento histórico da loucura, do discurso médico/ psiquiátrico e da identidade feminina, percebemos como esses são construções sociais. Ademais, ao nos atentarmos para as diferenças socioeconômicas e culturais envolvidas no processo de adoecimento mental feminino, entendemos que as experiências não são monolíticas, não há uma mulher universal, mas sim diversas mulheres e diversas formas de adoecimento.

Entretanto, apesar da diversidade cultural e do fato de negarmos uma generalização tanto das mulheres quanto do adoecimento mental, fica saliente na nossa análise que as mulheres, não importa se da África, América, Ásia ou Oceania, encontram-se irmanadas nos mesmos problemas oriundos da hegemonia machista e das desigualdades sociais e raciais em seus efeitos para a compreensão da sua saúde. Assim, a maternidade, as tarefas domésticas e demais funções de cuidado (trabalhos assumidos pelas mulheres e que são mal/não remunerados) têm custado a saúde mental feminina e não têm sido encarados com a devida importância na compreensão do seu adoecimento e, sobretudo, no tratamento de tais males. Por isso nos parece tão reducionista atribuir o adoecimento mental feminino apenas à dimensão natural. Nesse sentido, entendemos que essencializar o feminino, interpretando funções construídas socialmente e cristalizando-as em "tarefas de mulheres", retroalimenta o adoecimento.

Cremos que o destaque dado aos fatores sócio-históricos do adoecimento (Zanello, 2014), em detrimento à perspectiva biológica do transtorno mental nas mulheres, representa um avanço a uma leitura essencialista de tal adoecimento. Todavia, uma mirada sócio-histórica pode também culminar em um processo de patologização das mazelas sociais, como acredita Zanello (2014). Isso posto, propomos uma visão dos transtornos mentais que considere as dimensões sociais envolvidas no adoecimento de maneira a construir um novo lugar social para os saberes $p s i$, com deslocamento do papel de controle social para uma reconfiguração de tais saberes como instrumentos de denúncias das mazelas sociais. Nesse sentido, acreditamos que tal reconfiguração permitiria compreender os transtornos mentais

Nova Perspectiva Sistêmica, v. 29, n. 67, p. 70-84, agosto 2020. 
como construídos socialmente, entendendo que as classificações e diagnósticos se movem ao ritmo das transformações sociais. Ademais, os saberes $p s i$, ao assumirem um posicionamento mais crítico frente ao contexto social, se aproximam da reestruturação político-social proposta pela Reforma Psiquiátrica Brasileira, que vai além da mera reformulação de estruturas de tratamento, demandando discursos críticos atualizados que orientem a prática profissional.

\section{REFERÊNCIAS}

Abrams, D., Nguyen, L. Try Murphy, J., Lee, Y., Tran, N. Kr, \&Wiljer, D. (2016). Perceptions and experiences of perinatal mental disorders in rural, predominantly ethnic minority communities in northern Vietnam.International Journal of Mental Health Systems, 10(10), 1-10. http://doi.org/10.1186/s13033-016-0043-0

Alves, F. J., Machado, D. B., \& Barreto, M. L. (2018). Effect of the Brazilian cash transfer programme on suicide rates: a longitudinal analysis of the Brazilian municipalities. Social Psychiatry and Psychiatric Epidemiology, 54(5), 599-606. https://doi.org/10.1007/s00127-018-1627-6

Baére, F., \& Zanello, V. (2018). O gênero no comportamento suicida: uma leitura epidemiológica dos dados do Distrito Federal. Estudos de Psicologia, 23(2), 168178. https://doi.org/10.22491/1678-4669.20180017

Barata, R. B. (2009). Como e por que as desigualdades sociais fazem mal à saúde. Rio de Janeiro: Editora Fiocruz.

Bertolote, J. M. (2012). O Suicídio e sua Prevenção. São Paulo, SP: Editora Unesp.

Botelho, L. L. R., Cunha, C. C. de A.s \& Macedo, M. (2011). O método da revisão integrativa nos estudos organizacionais. Gestão e Sociedade, 5(11), 121-136.

Chojenta, C. L., Lucke, J. C., Forder, P. M., \& Loxton, D. J. (2016). Maternal health factors as risks for postnatal depression: a prospective longitudinal study. PLoS ONE, 11(1), 1-9. http://doi.org/10.1371/journal.pone.0147246

Chojenta, C., Harris, S., Reilly, N., Forder, Pry Austin, M. P.y \& Loxton, D. (2014). History of pregnancy loss increases the risk of mental health problems in subsequent pregnancies but not in the postpartum. PLOS ONE, 9(4), 1-7. http://doi. org/10.1371/journal.pone.0095038

Cunha, A. C. B., Pereira Junior, J. P., Caldeira, C. L. V., \& Carneiro, V. M. S. de P. (2016). Diagnóstico de malformações congênitas: impactos sobre a saúde mental de gestantes. Estud. Psicol. (Campinas), 33(4), 601-611. http://doi. org/10.1590/1982-02752016000400004

Del Priore, M. (2008). Magia e medicina na Colônia: o corpo feminino. In M. Del Priore (Org.), História das Mulheres no Brasil (pp. 78-114).São Paulo: Contexto.

Engel, M. (2008). Psiquiatria e feminilidade. In M. Del Priori (Org.), História das mulheres no Brasil (pp. 322-361). São Paulo: Contexto.

Gergen, K. (2010). El asedio del yo. In K. Gergen, El yo saturado: dilemas de identidad en el mundo contemporáneo (pp. 19-40). Barcelona: Paidós.

Gergen, K. (2011). Realidades y relaciones: aproximaciones a la construcción social. Barcelona: Paidós.

Ghaffar, R., Iqlbal, Q., Khalid, A., Saleem, Fr, Hassali, M. A., Baloch, N. S., Ahmad, F. D., Bashir, S., Haider, S., \&Bashaar, M. (2017). Frequency and predictors of anxiety and depression among pregnant women attending tertiary healthcare institutes of Quetta City, Pakistan.BMC Women's Health, 17(51), 1-8. http://doi.org/10.1186/ $\underline{\text { s12905-017-0411-1 }}$ 
Goes, E. F. \&Nascimento, E. R. (2013).Mulheres negras e brancas e os níveis de acesso aos serviços preventivos de saúde: uma análise sobre as desigualdades. Saúde em Debate, 37(99), 571-579.

Guadalupe, A. R., López Jiménez, J. L., \& García González, C. (2017). Climaterio, salud y depresión, un abordaje psicosocial: estudio exploratorio en un grupo de mujeres de la Ciudad de México. Revista Kairós Gerontologia, 20(1), 9-23.

Kang, Y., Yao, Y, Dou, J., Guo, X., Li, S., Zhao, C., Han, H., \& Li, B. (2016). Prevalence and risk factors of maternal anxiety in late pregnancy in China. International Journal of Environmental Research and Public Health, 13(468), 1-11. http://doi. org/10.3390/ijerph13050468

Kathree, T., Selohilwe, 0. M., Bhana, A., \& Petersen, I. (2014). Perceptions of postnatal depression and health care needs in a South African sample: The "mental" in maternal health care. BMC Women's Health, 14(140), 1-11.

Lima, J. D. (2016). Anarcha, Lucy e Betsy: as escravas mães da ginecologia moderna. Nexo. Acesso em https://www.nexojornal.com.br/expresso/2016/12/03/ Anarcha-Lucy-e-Betsy-as-escravas-m\%C3\%A3es-da-ginecologia-moderna

Lucchese, R., Simões, N. D., Monteiro, L. H. B., Vera, I., Fernandes, I. L., Castro, P. A., Silva, G. C., Evangelista, R. A., Bueno, A. A.s \& Lemos, M. F. (2017). Fatores associados à probabilidade de transtorno mental comum em gestante: estudo transversal. Escola Anna Nery, 21(3), 1-6. http://doi.org/10.1590/2177-9465-ean-2016-0094

Ludermir', A. B. (2008). Desigualdades de Classe, Gênero e Saúde Mental nas Cidades. PHYSIS Revista de Saúde Coletiva, 18(3), 451-467. http://doi.org/10.1590/ S0103-73312008000300005

Manual diagnóstico e estatístico de transtornos mentais [recurso eletrônico]: DSM5 / American Psychiatric Association; tradução: Maria Inês Corrêa Nascimento ... et al.]; revisão técnica: Aristides Volpato Cordioli ...[et al.]. - 5. ed. - Dados eletrônicos. - Porto Alegre:Artmed, 2014.

Miller, L. J., \& Ghadiali, N. Y. (2015). Gender-specific mental health care needs of women veterans treated for psychiatric disorders in a Veterans Administration Women's Health Clinic. Medical Care, 53(4Supplement 1), S93-S96.

Moraes, J. F. Q., Costa, A. B., Silva, F. T. R., Silva, N. M. M. G., \& Melo, S. C. C. S. (2017). Prevalence of depressive symptoms among pregnant adolescents. Journal of Nursing and Health, 7(1), 50-57.

Moura, V. F. S., Pedrão, L. J., Souza, A. C. S., \& Boaventura, R. P. (2015). A depressão em gestantes no final da gestação. Revista Eletrônica Saúde Mental Álcool Drog., 11(4), 234-242.

Nguyen, T. T., Tran, T: D., Tran, Tr, La, B., Nguyen, H., \& Fisher, J. (2015). Postpartum change in common mental disorders among rural Vietnamese women: incidence, recovery and risk and protective factors. British Journal of Psychiatry, 206(2), $110-115$.

Nogueira, C. (2001). Feminismo e Discurso do Género na Psicologia Social. Psicologia e Sociedade, (13), 1-28. http://doi.org/10.1590/S0102-37722008000200014

Nogueira, C. (2017). Interseccionalidade e Psicologia Feminista. Salvador: EditoraDevires.

Ogbo, F. A.s Eastwood, J., Hendry, A., Jalaludin, B., Agho, K. E., Barnett, B., \& Page, A. (2018).Determinants of antenatal depression and postnatal depression in Australia. BMC Psychiatry, 18(1), 1-11.

Onah, M. N., Field, S., Bantjes, J., \&Honikman, S. (2017). Perinatal suicidal ideation and behaviour: psychiatry and adversity. Archives of Women's Mental Health, 20(2), 321-331. 
Pereira, M. 0.,\& Amarante, P. Mulheres, loucura e patologização: desafios para a luta antimanicomial e a Reforma Psiquiátrica Brasileira. In R. G. Passos, R. A. Costa, \& F. G. Silva. Saúde mental e os desafios atuais da Atenção Psicossocial (pp. 159182). Rio de Janeiro: Gramma, 2017.

Reis, L. T., Bernardes, L. S., Barboza, M. C. N., \& Gonçalves, A. M. de S. (2015). Rastreamento de sintomas psiquiátricos não-psicóticos entre gestantes de um município do estado de Mato Grosso. Journal of Nursing and Health, 5(2), 141-152.

Spink, M. J. (2003). As origens históricas da obstetrícia moderna. In M. J. Spink, Psicologia social e saúde: práticas, saberes e sentidos (pp. 169-193). Petrópolis: Vozes.

Takahashi, Y., \&Tamakoshi, K. (2014). Factors associated with early postpartum maternity blues and depression tendency among japanese mothers with full-term healthy infants. Nagoya Journal of Medical Science, 76(1-2), 129-138.

Tol, W. A., Ebrecht, B., Aiyo, R., Murray, S. M.s Nguyen, A. J., Kohrt, B. A., Ndyanabangi, S.,Alderman, S., Musisi, S, \&Nakku, J. (2018). Maternal mental health priorities, help-seeking behaviors, and resources in post-conflict settings: a qualitative study in eastern Uganda. BMC Psychiatry, 18(39), 1-15.

Van Vo, T., Hoa, T. K. D., \& Hoang, T. D. (2017). Postpartum depressive symptoms and associated factors in married women: a cross-sectional study in Danang City, Vietnam. Frontiers in Public Health, 5, 1-8. http://doi.org/10.3389/ fpubh.2017.00093

Vázquez Sixto, F. (1996). El análisis de contenido temático. Objetivos y medios en la investigación psicosocial (Documento de trabajo) (pp. 47-70). Universitat Autònoma de Barcelona.

Verbeek, T., Arjadi, R., Vendrik, J. J., Burger, H., \& Berger, M. Y. (2015). Anxiety and depression during pregnancy in Central America: a cross-sectional study among pregnant women in the developing country Nicaragua. BMC Psychiatry, 15(292), 1-6. http://doi.org/10.1186/s12888-015-0671-y

Vieira, E. M. (1999). A medicalização do corpo feminino. In K. Giffin, \& S. H. Costa (Orgs.), Questões da Saúde Reprodutiva (pp. 67-78). Rio de Janeiro: Editora Fiocruz.

Wang, Y., Wang, X., Liu, Fu, Jiang, Xn, Xiao, Y., Dong, X., Kong, X., Yang, X., Tian, D., \&Qu, $Z_{\text {. }}$ (2016). Negative life events and antenatal depression among pregnant women in rural China: the role of negative automatic thoughts. PLoS ONE, 11(12), 1-14. http://doi.org/10.1371/journal.pone.0167597

Woolhouse, H., Gartland, D., Mensah, F., \& Brown, S. J. (2014). Maternal depression from early pregnancy to 4 years postpartum in a prospective pregnancy cohort study: Implications for primary health care. BJOG: An International Journal of Obstetrics and Gynecology, 122(3), 312-321. http://doi.org/10.1111/1471-0528.12837

Zanello, V. (2014). A saúde mental sob o viés do gênero: uma releitura gendrada da epidemiologia, da semiologia e da interpretação diagnóstica. In V. Zanello, \& A. P. M. Andrade (Orgs.), Saúde mental e gênero: diálogos, práticas e interdisciplinaridade (pp. 41-58). Curitiba: Appris. 


\section{AGRADECIMENTOS}

Agradecemos à Coordenação de Aperfeiçoamento de Pessoal de Nível Superior (CAPES) pela bolsa de Doutorado concedida.

\section{ANA CAROLINA CERQUEIRA MEDRADO}

Doutoranda do Programa de Pós-Graduação em Psicologia da UFBA. Psicóloga. Mestra em Saúde, Ambiente e Trabalho pela Faculdade de Medicina da Bahia (UFBA). Especialista em Saúde Mental na modalidade resi-dência multiprofissional em saúde (UNEB). Pesquisadora do Grupo de Pesquisa Laboratório de Estudos Vinculares em Saúde Mental do Instituto de Psicologia (UFBA).

https://orcid.org/0000-0002-0266-9360

E-mail: accm100@yahoo.com.br

\section{MÔNICA LIMA}

Professora Associada III da UFBA. Psicóloga. Possui Mestrado e Doutorado em Saúde Pública/Coletiva pelo Instituto de Saúde Coletiva (UFBA), Pós-Doutorado em Psicologia Social pela Universidad Autónoma de Barcelona (UAB). Pesquisadora do Grupo de Pesquisa Laboratório de Estudos Vinculares em Saúde Mental do IPS/ UFBA. Colaboradora do grupo de pesquisa "Laicos IAPSE" (UAB). Professora permanente da Pós-Graduação em Psicologia (IPS/UFBA).

https://orcid.org/0000-0002-6666-8463

E-mail: molije@hotmail.com 